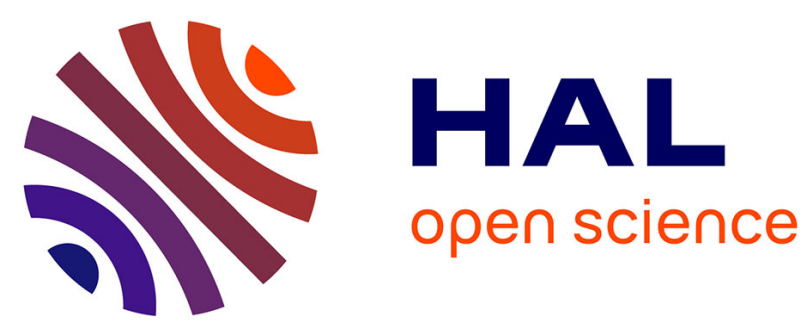

\title{
Isomerization of Chiral Non-Racemic $\alpha$-Substituted Propargylic Amines to Terminal Acetylenes
}

Jérôme Blanchet, Martine Bonin, Laurent Micouin, Henri-Philippe Husson

\section{To cite this version:}

Jérôme Blanchet, Martine Bonin, Laurent Micouin, Henri-Philippe Husson. Isomerization of Chiral Non-Racemic $\alpha$-Substituted Propargylic Amines to Terminal Acetylenes. European Journal of Organic Chemistry, 2002, 2002 (15), pp.2598-2602. 10.1002/1099-0690(200208)2002:153.0.CO;2-V . hal-02185592

\section{HAL Id: hal-02185592 \\ https://hal.science/hal-02185592}

Submitted on 16 Jul 2019

HAL is a multi-disciplinary open access archive for the deposit and dissemination of scientific research documents, whether they are published or not. The documents may come from teaching and research institutions in France or abroad, or from public or private research centers.
L'archive ouverte pluridisciplinaire HAL, est destinée au dépôt et à la diffusion de documents scientifiques de niveau recherche, publiés ou non, émanant des établissements d'enseignement et de recherche français ou étrangers, des laboratoires publics ou privés. 


\title{
Isomerization of chiral non-racemic $\alpha$-substituted propargylic amines to terminal acetylenes
}

\author{
J. Blanchet, M. Bonin, * L. Micouin,* and H.-P. Husson \\ Laboratoire de Chimie Thérapeutique associé au CNRS et à l'Université René Descartes (UMR \\ 8638), Faculté des Sciences Pharmaceutiques et Biologiques, \\ 4, av. de l'Observatoire, 75270 Paris cedex 06, France \\ Fax: (33) 0143291403 \\ E-mail: micouin@pharmacie.univ-paris5.fr, Bonin@pharmacie.univ-paris5.fr
}

\section{Received}

KAPA / isomerization / propargylic amines / phenylglycinol / alkyne

Various $\alpha$-substituted propargylamines prepared in three steps from $(R)$-phenylglycinol are readily isomerized at $0{ }^{\circ} \mathrm{C}$ with KAPA to terminal acetylenic amines, without any detectable epimerization of the chiral center, as already observed for propargylalcohols. Enantiomerically pure primary $\alpha-$ substituted alkynylamines can be easily obtained in two steps after removal of ferrocenylmethyl protective group and oxidative cleavage of the chiral appendage. 


\section{Introduction}

While $\omega$-propargylic alcohols are common synthetic intermediates in natural products synthesis, their nitrogenated counterparts have been less studied. ${ }^{[1]}$ The lack of a simple and straighforward $\omega$ propargylic amines preparation is probably one of the reasons for this fact.

One of the simpliest way to synthesize alcohols bearing a terminal acetylenic function is the isomerization of internal triple bonds to terminal acetylenes with potassium 3-aminopropylamide (KAPA). ${ }^{[2]}$ Furthermore, the use of this "acetylene zipper" with an optically-active secondary propargylic alcohol enables the rapid isomerization of the acetylene unit away from the hydroxy center, without racemization. ${ }^{[3]}$ Although the usefulness of this reaction has been proved both in several natural product total syntheses ${ }^{[4]}$ and for the efficient preparation of polydeuterated long chain derivatives, ${ }^{[5]}$ it has been mainly carried out only with dialkylacetylenes or alcohols.

We recently described a new access to enantiomerically pure $\alpha$-substituted propargylic amines via the nucleophilic opening of chiral non racemic oxazolidines with mixed organoaluminum reagents. ${ }^{[6]}$ We report in this communication that the isomerization of chiral $\alpha$-substituted propargylic amines to terminal acetylenic compounds by KAPA proceeds in efficient manner, without racemization.

\section{Results and Discussion}

Chiral non-racemic $\alpha$-substituted amino alcohols 4a-c were prepared from $(R)$-phenylglycinol 1 using our previously reported procedures (Scheme 1).

\section{((Scheme 1))}

The clean preparation of KAPA was performed according to the procedure described by Cossy and co-workers. ${ }^{[7]}$ Best results were obtained when this reagent was freshly prepared and immediately used. The isomerization reaction was carried out in 1,3-diaminopropane as solvent at $0{ }^{\circ} \mathrm{C}$ (Scheme 2). Terminal acetylenic amino alcohols 5a-c were obtained in less than $30 \mathrm{~min}$, in moderate to good yields, without any detectable epimerization. ${ }^{[8]}$

\section{((Scheme 2))}

Final deprotection leading to the primary amines was performed using oxidative cleavage of the amino alcohols. ${ }^{[6],[9],[10]}$ Although these compounds proved to be more stable than the previously 
described $\alpha$-substituted propargylamines, they were hygroscopic and therefore protected and stored as benzamides 7a-c (Scheme 3).

\section{$(($ Scheme 3))}

All the attempts to isomerize N-protected compounds 3a-c led to extensive degradation, probably because of the base sensitive ferrocenyl moiety. ${ }^{[11]}$ The presence of the free hydroxyl group proved to be essential to achieve completion of the reaction. Indeed, when submitted to KAPA isomerization, O-protected compound $\mathbf{8}$ led to a complex mixture of by-products, among which rearranged derivative 9 could be separated and characterized in less than $20 \%$ yield (Scheme 4$).{ }^{[12]}$

\section{$(($ Scheme 4))}

In the case of propargyl alcohols isomerization, the absence of racemization was explained by the quantitative formation of the negatively charged alkoxide, leading to a decrease of the acidity of the neighboring protons, and therefore to the protection of the base sensitive stereogenic center. In this study, the formation of the potassium alkoxide avoids epimerization and degradation by protecting base sensitive protons $\alpha$ to the nitrogen atom, probably via the formation of a five membered chelate $\mathbf{1 0}$ with nitrogen (Scheme 5).

\section{$(($ Scheme 5))}

\section{Conclusion}

We have described a new application of KAPA induced isomerization, leading in a simple and efficient way to optically pure $\alpha$-substituted amines bearing a terminal acetylenic function. These compounds can be useful synthetic intermediates for the elaboration of cyclic or acyclic enantiopure amino derivatives.

\section{Experimental Section}

\section{General Procedures.}

NMR spectra were obtained at $300 \mathrm{MHz}\left({ }^{1} \mathrm{H}\right.$ field value) on a Bruker AC 300 . Routine infrared spectra (IR) were recorded neat on a Perkin-Elmer 1600 spectrophotometer as thin film unless otherwise stated. Optical rotation measurement were performed using a $1 \mathrm{dm}$ path length cell. Elemental analyses were obtained from the Service de microanalyse of the Institut de Chimie des 
Substances Naturelles, Gif-sur-Yvette, France. Product purification was performed by flash chromatography on silica gel (Merck, 230-400 mesh). Mass spectra data were recorded in the chemical-ionisation mode $\left(\mathrm{NH}_{3}\right)$ on a AEI MS-9 spectrometer. High-resolution mass spectra were obtained on a Kratos MS 80RF spectrometer.

\section{General procedure for the preparation of propargylamines 3.}

The preparation of compound $\mathbf{3 b}$ is representative. To a DIBAL solution (1 $\mathrm{M}$ in toluene, $6.3 \mathrm{~mL}$, $6.3 \mathrm{mmol}$ ) under argon was slowly added freshly distilled triethylamine (920 $\mu \mathrm{L}, 6.6 \mathrm{mmol})$. The solution was stirred for $15 \mathrm{~min}$, cooled to $0{ }^{\circ} \mathrm{C}$ and heptyne $(1.6 \mathrm{~mL}, 12.2 \mathrm{mmol})$ was added dropwise. The reaction mixture was allowed to reach room temperature and stirred until hydrogen evolution stopped, cooled to $0{ }^{\circ} \mathrm{C}$ and a solution of oxazolidine $2 \mathbf{b}(1.17 \mathrm{~g}, 3.0 \mathrm{mmol})$ in toluene (10 $\mathrm{mL}$ ) was slowly added over $30 \mathrm{~min}$, followed by $\mathrm{Me}_{3} \mathrm{Al}$ solution ( $2 \mathrm{M}$ in heptane, $3.3 \mathrm{~mL}, 6.6$ $\mathrm{mmol}$ ). The solution was stirred for another $30 \mathrm{~min}$ at $0{ }^{\circ} \mathrm{C}$ and allowed to reach room temperature. The mixture was slowly poured on a cold solution of saturated Rochelle's salts and after vigorous stirring, the aqueous layer was twice extracted with diethylether. Organic layer was dried over anhydrous $\mathrm{MgSO}_{4}$, solvent was evaporated and the crude residue was purified by column chromatography (silica gel : 9/1 cyclohexane/ethyl acetate) to give $\mathbf{3 b}(1.21 \mathrm{~g}, 2.37 \mathrm{mmol}, 83 \%$ from 1).

\section{(1R,2R)-2-[Ferrocenyl-methyl-(1-methyl-oct-2-ynyl)-amino]-2-phenyl-ethanol (3a)} red-brown oil. $-[\alpha]_{\mathrm{D}}=+8.8(c=1.0, \mathrm{MeOH}) ;{ }^{1} \mathrm{H} \mathrm{NMR}\left(300 \mathrm{MHz}, \mathrm{CDCl}_{3}\right): \delta=0.89(\mathrm{t}, J=7.1 \mathrm{~Hz}$, $3 \mathrm{H}), 1.12(\mathrm{~d}, J=6.9 \mathrm{~Hz}, 3 \mathrm{H}), 1.30-1.43(\mathrm{~m}, 4 \mathrm{H}), 1.44-1.56(\mathrm{~m}, 2 \mathrm{H}), 2.19(\mathrm{td}, J=7.1,1.9 \mathrm{~Hz}, 2 \mathrm{H}), 2.71$ (br. s, 1H), 3.59 (d, $J=14.1 \mathrm{~Hz}, 1 \mathrm{H}), 3.75$ (d, $J=14.1 \mathrm{~Hz}, 1 \mathrm{H}), 3.78-3.89$ (m, 3H), 3.96-4.14 (m, 5H), $4.09(\mathrm{~s}, 5 \mathrm{H}), 4.19(\mathrm{~d}, J=11.6 \mathrm{~Hz}, 2 \mathrm{H}), 7.35-7.24(\mathrm{~m}, 5 \mathrm{H}) ;{ }^{13} \mathrm{C} \mathrm{NMR}\left(75 \mathrm{MHz}, \mathrm{CDCl}_{3}\right) \delta=14.0,18.5$, 20.9, 22.0, 28.4, 30.9, 45.3, 46.5, 61.4, 64.0, 67.6-69.6, 81.2, 84.6, 86.3, 127.1-128.4, 139.7; IR (cm-1, neat) : 3452, 3090, 2928, 2859, 1601. MS (CI:NH 3$): m / z=458\left[\mathrm{MH}^{+}\right]$. - Anal. Calcd for $\mathrm{C}_{28} \mathrm{H}_{35} \mathrm{NOFe}$ : C 73.52, H 7.71, N 3.06. Found: C 73.33, H 7.84, N 3.02.

\section{(1R,2R)-2-[Ferrocenyl-methyl-(1-isopropyl-oct-2-ynyl)-amino]-2-phenyl-ethanol (3b)}

orange oil. $-[\alpha] \mathrm{D}=+48\left(c 1.2, \mathrm{CHCl}_{3}\right) ;{ }^{1} \mathrm{H} \mathrm{NMR}\left(300 \mathrm{MHz}, \mathrm{CDCl}_{3}\right): \delta=0.64(\mathrm{~d}, J=6.5 \mathrm{~Hz}$, $3 \mathrm{H}), 0.91(\mathrm{~m}, 6 \mathrm{H}), 1.36(\mathrm{~m}, 4 \mathrm{H}), 1.48(\mathrm{~m}, 2 \mathrm{H}), 1.67(\mathrm{~m}, 1 \mathrm{H}), 2.19(\mathrm{td}, J=7.0,1.7 \mathrm{~Hz}, 2 \mathrm{H}), 2.50$ (br. s., $1 \mathrm{H}), 3.02$ (dd, $J=10.0,1.4 \mathrm{~Hz}, 1 \mathrm{H}), 3.65$ (d, $J=14.0 \mathrm{~Hz}, 1 \mathrm{H}), 3.72$ (d, $J=14.0 \mathrm{~Hz}, 1 \mathrm{H})$, $3.98(\mathrm{~m}, 2 \mathrm{H}), 4.13(\mathrm{~m}, 9 \mathrm{H}), 7.22(\mathrm{~m}, 5 \mathrm{H}) ;{ }^{13} \mathrm{C} \mathrm{NMR}\left(75 \mathrm{MHz}, \mathrm{CDCl}_{3}\right) \delta=14.0,18.6,19.9,20.5$, 22.1, 28.6, 31.0, 32.2, 46.4, 59.0, 61.7, 64.9, 67.4, 70.3, 86.3, 127.0, 127.8, 128.8, 139.7; IR (cm-1, 
neat) : 3427, 3085, 1598, 1490. MS (CI:NH$): m / z=486\left[\mathrm{MH}^{+}\right]$. - Anal. Calcd for $\mathrm{C}_{30} \mathrm{H} 39 \mathrm{FeNO}$ :

C, 74.22; H, 8.10; N, 2.89. Found: C, 73.97; H, 8.35; N, 2.79.

\section{(1R,2R)-2-[Ferrocenyl-methyl-(1-phenethyl-oct-2-ynyl)-amino]-2-phenyl-ethanol (3c)}

red-brown oil. $-[\alpha] \mathrm{D}=+9.4\left(c=1.1, \mathrm{CHCl}_{3}\right) ;{ }^{1} \mathrm{H} \mathrm{NMR}\left(300 \mathrm{MHz}, \mathrm{CDCl}_{3}\right): \delta=0.91(\mathrm{t}, J=7.0 \mathrm{~Hz}, 3 \mathrm{H})$, $1.32-1.40(\mathrm{~m}, 4 \mathrm{H}), 1.44-1.53(\mathrm{~m}, 2 \mathrm{H}), 1.64-1.76(\mathrm{~m}, 2 \mathrm{H}), 2.21(\mathrm{dt}, J=6.9,2.0 \mathrm{~Hz}, 2 \mathrm{H}), 2.43-2.50(\mathrm{~m}$, 2H), 2.59 (br. s, $1 \mathrm{H}), 3.53-3.61(\mathrm{~m}, 1 \mathrm{H}), 3.63(\mathrm{~d}, J=14.0 \mathrm{~Hz}, 1 \mathrm{H}), 3.77$ (d, $J=14.0 \mathrm{~Hz}, 1 \mathrm{H}), 3.82-3.92$ $(\mathrm{m}, 1 \mathrm{H}), 3.98-4.09(\mathrm{~m}, 4 \mathrm{H}), 4.07(\mathrm{~s}, 5 \mathrm{H}), 4.11-4.13(\mathrm{~m}, 1 \mathrm{H}), 4.20-4.22(\mathrm{~m}, 1 \mathrm{H}), 6.96-7.29(\mathrm{~m}, 10 \mathrm{H}) .{ }^{13} \mathrm{C}$ NMR $\left(75 \mathrm{MHz}, \mathrm{CDCl}_{3}\right) \delta=14.0,18.6,22.1,28.5,31.0,32.7,36.6,45.9,51.2,61.5,64.5,67.8,68.4$, 69.6, 69.9, 80.1, 85.8, 86.3, 125.6, 127.2, 128.3-129.1, 139.6 141.7. IR (cm-1, neat): 3446 ; $3086 ; 3027$; $2926 ; 2858 ; 1602 ; 1495 ; 1454 ; 1266 ; 1231 ; 1106 ; 1024 . \mathrm{m} / z=548\left[\mathrm{MH}^{+}\right]$. - Anal. Calcd for $\mathrm{C}_{35} \mathrm{H}_{41} \mathrm{NOFe}: \mathrm{C} 76.77, \mathrm{H} 7.55, \mathrm{~N} 2.56$. Found: C 76.47, H 7.36, N 2.57.

\section{General procedure for the preparation of propargylamines 4 .}

The preparation of compound $\mathbf{4 b}$ is representative. To a cold $\left(0{ }^{\circ} \mathrm{C}\right)$ solution of propargylamine $\mathbf{3 b}$ $(1.45 \mathrm{~g}, 3.0 \mathrm{mmol})$ in dry dichloromethane $(10 \mathrm{~mL})$ was added successively triethylsilane $(1.2 \mathrm{~mL}$, $7.5 \mathrm{mmol})$ and a solution of trifluoroacetic acid $(1.2 \mathrm{~mL}, 15 \mathrm{mmol})$ in dichloromethane $(15 \mathrm{~mL})$. The reaction mixture was allowed to reach room temperature, stirred for $2 \mathrm{~h}$ and quenched with water and saturated aqueous solution of $\mathrm{K}_{2} \mathrm{CO}_{3}$. Aqueous layer was extracted twice with dichloromethane, the organic layer was dried over anhydrous $\mathrm{MgSO}_{4}$ and solvent was evaporated. The crude residue was purified by column chromatography (silica gel, two successive elutions : 9/1 cyclohexane/ethyl acetate then 1/1 cyclohexane/ethyl acetate) to give $\mathbf{4 b}$ (723 mg, $84 \%$ ).

\section{(1R,2R)-2-(1-Methyl-oct-2-ynylamino)-2-phenyl-ethanol (4a)}

oil. $-[\alpha] \mathrm{D}=-13.6(c=1.25, \mathrm{MeOH}) ;{ }^{1} \mathrm{H} \mathrm{NMR}\left(300 \mathrm{MHz}, \mathrm{CDCl}_{3}\right): \delta=0.88(\mathrm{t}, J=7.0 \mathrm{~Hz}, 3 \mathrm{H}), 1.28(\mathrm{~d}$, $J=6.6 \mathrm{~Hz}, 3 \mathrm{H}), 1.28-1.37(\mathrm{~m}, 4 \mathrm{H}), 1.38-1.46(\mathrm{~m}, 2 \mathrm{H}), 2.08(\mathrm{td}, J=6.9,2.0 \mathrm{~Hz}, 2 \mathrm{H}), 2.48$ (br. s, 2H), $3.52(\mathrm{qt}, J=6.6,2.0 \mathrm{~Hz}, 1 \mathrm{H}), 3.57(\mathrm{dd}, J=10.9,6.7 \mathrm{~Hz}, 1 \mathrm{H}), 3.71(\mathrm{dd}, J=10.9,4.7 \mathrm{~Hz}, 1 \mathrm{H}), 3.98(\mathrm{dd}, J$ $=6.7,4.7 \mathrm{~Hz}, 1 \mathrm{H}), 7.24-7.35(\mathrm{~m}, 5 \mathrm{H}) .-{ }^{13} \mathrm{C} \mathrm{NMR}\left(75 \mathrm{MHz}, \mathrm{CDCl}_{3}\right) \delta=13.9,18.5,22.0,22.4,28.4$, 30.9, 43.0, 61.4, 65.4, 82.2, 83.1, 127.2-128.4, 141.1. - IR ( $\mathrm{cm}^{-1}$, neat): 3330, 2931, 2859, 1603. - MS $\left(\mathrm{CI}: \mathrm{NH}_{3}\right): m / z=260\left[\mathrm{MH}^{+}\right]$. - Anal. Calcd for $\mathrm{C}_{17} \mathrm{H}_{25} \mathrm{NO}, 1 / 2 \mathrm{H}_{2} \mathrm{O}: \mathrm{C} 76.08, \mathrm{H}$ 9.71, N 5.22. Found: C 76.48, H 9.35, N 5.03. 


\section{(1R,2R)-2-(1-isopropyl-oct-2-ynylamino)-2-phenyl-ethanol (4b)}

oil. $-[\alpha]_{\mathrm{D}}=-13\left(c\right.$ 1.15, $\left.\mathrm{CHCl}_{3}\right) .{ }^{1} \mathrm{H}$ NMR $\left(300 \mathrm{MHz}, \mathrm{CDCl}_{3}\right): \delta=0.88(\mathrm{t}, J=7.0 \mathrm{~Hz}, 3 \mathrm{H}), 0.97$ $(\mathrm{d}, J=6.6 \mathrm{~Hz}, 3 \mathrm{H}), 0.99(\mathrm{~d}, J=6.6 \mathrm{~Hz}, 3 \mathrm{H}), 1.31(\mathrm{~m}, 4 \mathrm{H}), 1.42(\mathrm{~m}, 2 \mathrm{H}), 1.83(\mathrm{~m}, 1 \mathrm{H}), 2.11(\mathrm{td}, J=$ 6.6, 2.6 Hz, 2H), $3.22(\mathrm{~m}, 1 \mathrm{H}), 3.53(\mathrm{dd}, J=10.7,6.2 \mathrm{~Hz}, 1 \mathrm{H}), 3.72(\mathrm{dd}, J=10.7,4.7 \mathrm{~Hz}, 1 \mathrm{H}), 3.99$ $(\mathrm{dd}, J=6.2,4.7 \mathrm{~Hz}, 1 \mathrm{H}), 7.29(\mathrm{~m}, 5 \mathrm{H}) .-{ }^{13} \mathrm{C} \mathrm{NMR}\left(75 \mathrm{MHz}, \mathrm{CDCl}_{3}\right) \delta=13.9,17.4,18.5,19.6$, 22.0, 28.4, 30.9, 32.2, 54.3, 61.8, 65.1, 79.7, 84.8, 127.3-128.5, 141.6. - IR (cm-1, neat) : 3349, 2930, 2871, 2244. - MS (CI:NH $): m / z=288\left[\mathrm{MH}^{+}\right]$.

\section{(1R,2R)-2-(1-Phenethyl-oct-2-ynylamino)-2-phenyl-ethanol (4c)}

oil. $-[\alpha] \mathrm{D}=-57.5\left(c=0.8, \mathrm{CHCl}_{3}\right) ;{ }^{1} \mathrm{H} \mathrm{NMR}\left(300 \mathrm{MHz}, \mathrm{CDCl}_{3}\right): \delta=0.89(\mathrm{t}, J=7.0 \mathrm{~Hz}, 3 \mathrm{H})$, 1.27-1.39 (m, 4H), 1.39-1.48 (m, 2H), 1.78-2.02 (m, 2H), $2.11(\mathrm{td}, J=6.9,2.0 \mathrm{~Hz}, 2 \mathrm{H}), 2.12(\mathrm{~m}$, 2H), 2.67-2.88 (m, 2H), $3.40(\mathrm{ddt}, J=7.7,5.7,2.0 \mathrm{~Hz}, 1 \mathrm{H}), 3.54(\mathrm{dd}, J=10.8,6.6 \mathrm{~Hz}, 1 \mathrm{H}), 3.70$ (dd, $J=10.8,4.7 \mathrm{~Hz}, 1 \mathrm{H}), 3.97(\mathrm{dd}, J=6.6,4.7 \mathrm{~Hz}, 1 \mathrm{H}), 7.15-7.34(\mathrm{~m}, 10 \mathrm{H}) .-{ }^{13} \mathrm{C}$ NMR $(75$ $\left.\mathrm{MHz}, \mathrm{CDCl}_{3}\right) \delta=14.0,18.6,22.2,28.4,31.0,32.1,37.7,47.8,61.6,65.3,81.0,84.6,125.8-128.4$, 141.4, 141.7. - IR (cm-1, neat): 3321, 3026, 2930, 2859, 1603, 1495, 1454, 1330, 1107, 1030. - MS $\left(\mathrm{CI}: \mathrm{NH}_{3}\right): m / z=350\left[\mathrm{MH}^{+}\right]$. - Anal. Calcd for $\mathrm{C}_{2} 4 \mathrm{H}_{3} 1 \mathrm{NO}, 1 / 4 \mathrm{H}_{2} \mathrm{O}: \mathrm{C} 80.89, \mathrm{H} 8.76, \mathrm{~N} 4.10$. Found: C 80.69, H 8.58, N 3.77.

\section{General procedure for the isomerization of propargylamines 4.}

The preparation of compound $\mathbf{5 b}$ is representative. 1,3-diaminopropane $(8 \mathrm{~mL})$ was added to potassium hydride $(328 \mathrm{mg}, 8.2 \mathrm{mmol})$ at $0{ }^{\circ} \mathrm{C}$ under argon. The suspension was stirred at room temperature for $30 \mathrm{~min}$ and cooled to $0{ }^{\circ} \mathrm{C}$. Propargylamine $4 \mathbf{b}(470 \mathrm{mg}, 1.64 \mathrm{mmol})$ in 1,3diaminopropane $(2 \mathrm{~mL})$ was slowly added, the reaction mixture was stirred $30 \mathrm{~min}$ and treated with a saturated aqueous ammonium chloride solution $(10 \mathrm{~mL})$. The aqueous phase was extracted twice with ether, organic layers were washed with a saturated aqueous sodium chloride solution, dried over anhydrous $\mathrm{MgSO}_{4}$ and solvent was evaporated. The crude residue was purified by column chromatography (silica gel, 9/1 cyclohexane/ethyl acetate) to give $\mathbf{5 b}(291 \mathbf{m g}, 62 \%)$.

\section{(1R,2R)-2-(1-methyl-oct-7-ynylamino)-2-phenyl-ethanol (5a)}

oil. $-[\alpha]_{\mathrm{D}}=-59\left(c 1.15, \mathrm{CHCl}_{3}\right) .-{ }^{1} \mathrm{H} \mathrm{NMR}\left(300 \mathrm{MHz}, \mathrm{CDCl}_{3}\right): \delta=1.01(\mathrm{~d}, J=6.2 \mathrm{~Hz}, 3 \mathrm{H})$, $1.29(\mathrm{~m}, 6 \mathrm{H}), 1.47(\mathrm{~m}, 2 \mathrm{H}), 1.89(\mathrm{t}, J=2.6 \mathrm{~Hz}, 1 \mathrm{H}), 2.12(\mathrm{td}, J=6.9,2.6 \mathrm{~Hz}, 2 \mathrm{H}), 2.24(\mathrm{~m}, 1 \mathrm{H})$ 2.51 (br. s, 2H) 3.48 (dd, $J=10.6,8.6 \mathrm{~Hz}, 1 \mathrm{H}), 3.67$ (dd, $J=10.6,4.5 \mathrm{~Hz}, 1 \mathrm{H}), 3.87$ (dd, $J=8.6$, 
$4.5 \mathrm{~Hz}, 1 \mathrm{H}), 7.29(\mathrm{~m}, 5 \mathrm{H}) .-{ }^{13} \mathrm{C} \mathrm{NMR}\left(75 \mathrm{MHz}, \mathrm{CDCl}_{3}\right) \delta=18.2,19.8,25.3,28.2,28.6,37.8$, 49.3, 61.2, 66.7, 68.1, 84.5, 127.1, 127.3, 128.4, 141.1. IR ( $\mathrm{cm}^{-1}$, neat) : 3300, 2934, 2858. MS $\left(\mathrm{CI}: \mathrm{NH}_{3}\right): m / z=260\left[\mathrm{MH}^{+}\right]$. - Anal. Calcd for $\mathrm{C}_{17} \mathrm{H}_{25} \mathrm{NO}: \mathrm{C}, 78.72 ; \mathrm{H}, 9.71 ; \mathrm{N}, 5.40$. Found: C, 78.53; H, 9.87; N, 5.36.

\section{(1S,2R)-2-(1-isopropyl-oct-7-ynylamino)-2-phenyl-ethanol (5b)}

oil. $-[\alpha]_{\mathrm{D}}=-74\left(c 1.05, \mathrm{CHCl}_{3}\right) .-{ }^{1} \mathrm{H} \mathrm{NMR}\left(300 \mathrm{MHz}, \mathrm{CDCl}_{3}\right): \delta=0.81(\mathrm{~d}, J=6.7 \mathrm{~Hz}, 3 \mathrm{H})$, $0.88(\mathrm{~d}, J=6.7 \mathrm{~Hz}, 3 \mathrm{H}), 1.15(\mathrm{~m}, 6 \mathrm{H}), 1.38(\mathrm{~m}, 2 \mathrm{H}), 1.85(\mathrm{~m}, 1 \mathrm{H}), 1.90(\mathrm{t}, J=2.6 \mathrm{~Hz}, 1 \mathrm{H}), 2.06$ (td, $J=6.9,2.6 \mathrm{~Hz}, 2 \mathrm{H}), 2.18$ (br. s., $2 \mathrm{H}), 2.25$ (m, 1H) 3.47 (dd, $J=10.4,8.8 \mathrm{~Hz}, 1 \mathrm{H}), 3.64$ (dd, $J$ $=10.4,4.5 \mathrm{~Hz}, 1 \mathrm{H}), 3.78(\mathrm{dd}, J=8.8,4.5 \mathrm{~Hz}, 1 \mathrm{H}), 7.28(\mathrm{~m}, 5 \mathrm{H}) .-13 \mathrm{C} \mathrm{NMR}\left(75 \mathrm{MHz}, \mathrm{CDCl}_{3}\right) \delta=$ $16.8,18.2,18.7,25.7,28.2,28.6,29.2,29.9,59.2,62.1,66.4,68.1,84.5,127.2,127.4,128.4,141.5$; IR $\left(\mathrm{cm}^{-1}\right.$, neat) : 3307, 2935, 2863.- MS (CI:NH$): m / z=288\left[\mathrm{MH}^{+}\right]$. - Anal. Calcd for $\mathrm{C}_{19 \mathrm{H}} 29 \mathrm{NO}$ : C, 79.39; H, 10.17; N, 4.87. Found: C, 79.37; H, 10.29; N, 4.79.

\section{(1S,2R)-2-(1-phenethyl-oct-7-ynylamino)-2-phenyl-ethanol (5c)}

oil. $-[\alpha]_{\mathrm{D}}=-89\left(\mathrm{c} 1.15, \mathrm{CHCl}_{3}\right) .-{ }^{1} \mathrm{H} \mathrm{NMR}\left(300 \mathrm{MHz}, \mathrm{CDCl}_{3}\right): \delta=1.30(\mathrm{~m}, 8 \mathrm{H}), 1.71(\mathrm{~m}, 2 \mathrm{H})$, $1.92(\mathrm{t}, J=2.6 \mathrm{~Hz}, 1 \mathrm{H}), 2.18(\mathrm{td}, J=7.0,2.6 \mathrm{~Hz}, 2 \mathrm{H}), 2.24$ (br. s., $2 \mathrm{H}), 2.60$ (m, 3H) $3.49(\mathrm{dd}, J=$ 10.6, 8.7 Hz, 1H), $3.64(\mathrm{dd}, J=10.6,4.5 \mathrm{~Hz}, 1 \mathrm{H}), 3.79(\mathrm{dd}, J=8.7,4.5 \mathrm{~Hz}, 1 \mathrm{H}), 7.24(\mathrm{~m}, 10 \mathrm{H})$. -

${ }^{13} \mathrm{C} \mathrm{NMR}\left(75 \mathrm{MHz}, \mathrm{CDCl}_{3}\right) \delta=18.3,24.1,28.3,28.7,31.3,34.4,35.4,53.6,61.7,66.5,68.1$, 84.5, 125.6, 128.5, 141.3, 142.5. - IR $\left(\mathrm{cm}^{-1}\right.$, neat $): 3300,2932,2857,1602 . \mathrm{MS}\left(\mathrm{CI}: \mathrm{NH}_{3}\right): m / z=$ $350\left[\mathrm{MH}^{+}\right.$]. - Anal. Calcd for $\mathrm{C}_{24} \mathrm{H}_{31} \mathrm{NO}: \mathrm{C}, 82.47 ; \mathrm{H}, 8.94 ; \mathrm{N}, 4.01$. Found: C, 82.31; H, 9.13; N, 4.06 .

\section{General procedure for the oxidative cleavage.}

The preparation of compound $\mathbf{6 b}$ is representative. To a cold $\left(0^{\circ} \mathrm{C}\right)$ solution of propargylamine $\mathbf{5 b}$ (410 mg, $1.43 \mathrm{mmol})$ in methanol $(8.5 \mathrm{~mL})$ was added aqueous methylamine (40\% solution, $1 \mathrm{~mL})$ and slowly an aqueous solution of periodic acid $(845 \mathrm{mg}, 3.72 \mathrm{mmol}$ in $7.5 \mathrm{ml} \mathrm{H} 2 \mathrm{O}$ ). The turbid solution was stirred at room temperature for $3 \mathrm{~h}$ and became limpid. The reaction mixture was extracted twice with ether. After evaporation of ether, the methanolic solution was treated with $3 \mathrm{M}$ aqueous $\mathrm{HCl}$, methanol was evaporated and the aqueous phase was washed twice with ether. Then, the aqueous phase was treated with an aqueous saturated solution of $\mathrm{K}_{2} \mathrm{CO}_{3}$ to reach $\mathrm{pH} 9$ and extracted twice with dichloromethane. Organic layer was dried over anhydrous $\mathrm{MgSO}_{4}$ and the 
primary amine was purified by column chromatography (silica gel, ethyl acetate) to give $\mathbf{6 b}$ (222 mg, $93 \%)$.

\section{(R)-1-methyl-oct-7-ynylamine (6a)}

oil. $-[\alpha] \mathrm{D}=-8\left(c\right.$ 1.05, $\left.\mathrm{CHCl}_{3}\right) .{ }^{1} \mathrm{H} \mathrm{NMR}\left(300 \mathrm{MHz}, \mathrm{CDCl}_{3}\right): \delta=1.06(\mathrm{~d}, J=6.6 \mathrm{~Hz}, 3 \mathrm{H})$, $1.36(\mathrm{~m}, 6 \mathrm{H}), 1.53(\mathrm{~m}, 2 \mathrm{H}), 1.66$ (br. s, 2H), 1.93 (t, $J=2.6 \mathrm{~Hz}, 1 \mathrm{H}), 2.18$ (td, $J=6.6,2.6 \mathrm{~Hz}, 2 \mathrm{H})$, $2.87(\mathrm{~m}, 1 \mathrm{H}) .-{ }^{13} \mathrm{C} \mathrm{NMR}\left(75 \mathrm{MHz}, \mathrm{CDCl}_{3}\right) \delta=18.3,23.9,25.8,28.3,28.7,39.9,46.8,68.1$, 84.6. - IR $\left(\mathrm{cm}^{-1}\right.$, neat $): 3306,2931,2857,2116 .-\mathrm{MS}\left(\mathrm{CI}: \mathrm{NH}_{3}\right): \mathrm{m} / z=140\left(\mathrm{MH}^{+}\right)$.

\section{(S)-1-isopropyl-oct-7-ynylamine (6b)}

oil. $-[\alpha]_{\mathrm{D}}=-18\left(c 1.20, \mathrm{CHCl}_{3}\right) .-{ }^{1} \mathrm{H} \mathrm{NMR}\left(300 \mathrm{MHz}, \mathrm{CDCl}_{3}\right): \delta=0.87(\mathrm{~d}, J=6.8 \mathrm{~Hz}, 3 \mathrm{H})$, $0.92(\mathrm{~d}, J=6.8 \mathrm{~Hz}, 3 \mathrm{H}), 1.44(\mathrm{~m}, 11 \mathrm{H}), 1.92(\mathrm{t}, J=2.6 \mathrm{~Hz}, 1 \mathrm{H}), 2.19(\mathrm{td}, J=6.6,2.6 \mathrm{~Hz}, 2 \mathrm{H}), 2.52$ (m, 1H). - ${ }^{13} \mathrm{C} \mathrm{NMR}\left(75 \mathrm{MHz}, \mathrm{CDCl}_{3}\right) \delta=17.0,18.3,19.1,26.0,28.4,28.8,33.1,34.4,56.4,68.1$, 84.5. - IR $\left(\mathrm{cm}^{-1}\right.$, neat $): 3308,2933,2860,2116$. $\mathrm{MS}\left(\mathrm{CI}: \mathrm{NH}_{3}\right): \mathrm{m} / z=168\left(\mathrm{MH}^{+}\right)$.

\section{(S)-1-phenethyl-7-ynylamine (6c)}

amorphous $-[\alpha] \mathrm{D}=+5\left(c 1.05, \mathrm{CHCl}_{3}\right) .-{ }^{1} \mathrm{H} \mathrm{NMR}\left(300 \mathrm{MHz}, \mathrm{CDCl}_{3}\right): \delta=1.41(\mathrm{~m}, 11 \mathrm{H}), 1.72$ $(\mathrm{m}, 1 \mathrm{H}), 1.95(\mathrm{t}, J=2.6 \mathrm{~Hz}, 1 \mathrm{H}), 2.19(\mathrm{td}, J=7.0,2.6 \mathrm{~Hz}, 2 \mathrm{H}), 2.69(\mathrm{~m}, 3 \mathrm{H}), 7.17(\mathrm{~m}, 3 \mathrm{H}), 7.28$ (m, 2H). - ${ }^{13} \mathrm{C} \mathrm{NMR}\left(75 \mathrm{MHz}, \mathrm{CDCl}_{3}\right) \delta=18.3,25.5,28.3,28.7,32.5,37.9,39.8,50.7,68.1,84.5$, 125.6, 128.3, 142.3. - IR $\left(\mathrm{cm}^{-1}\right.$, neat $): 3303,3026,2931,2856,2116$. $-\mathrm{MS}\left(\mathrm{CI}: \mathrm{NH}_{3}\right): \mathrm{m} / z=230$ $\left(\mathrm{MH}^{+}\right)$.

\section{General procedure for preparation of benzamides 7.}

The preparation of compound $\mathbf{7 b}$ is representative. Freshly purified primary amine $\mathbf{6 b}(120 \mathrm{mg}$, $0.72 \mathrm{mmol})$ was dissolved in dichloromethane $(10 \mathrm{~mL})$. Benzoyl chloride (337 $\mu \mathrm{L}, 2.9 \mathrm{mmol})$ and triethylamine (407 $\mu \mathrm{L}, 2.9 \mathrm{mmol}$ ) were added and the reaction mixture was stirred overnight, washed with water. The organic layer was dried over anhydrous $\mathrm{MgSO}_{4}$ and evaporated. Crude residue was purified by column chromatography (silica gel, 7/3 cyclohexane/ethyl acetate) to give 7b (141 mg, $67 \%$ from 5b). 


\section{(R)-N-(1-methyl-oct-7-ynyl)-benzamide (7a)}

white solid. - M.p. $=57{ }^{\circ} \mathrm{C} .-[\alpha] \mathrm{D}=-13\left(c\right.$ 1.05, $\left.\mathrm{CHCl}_{3}\right) .-{ }^{1} \mathrm{H}$ NMR $\left(300 \mathrm{MHz}, \mathrm{CDCl}_{3}\right): \delta=$ $1.21(\mathrm{~d}, J=6.6 \mathrm{~Hz}, 3 \mathrm{H}), 1.44(\mathrm{~m}, 8 \mathrm{H}), 1.92(\mathrm{t}, J=2.6 \mathrm{~Hz}, 1 \mathrm{H}), 2.17(\mathrm{td}, J=6.9,2.6 \mathrm{~Hz}, 2 \mathrm{H}), 4.17$ $(\mathrm{m}, 1 \mathrm{H}) 6.28(\mathrm{~d}, J=8.0 \mathrm{~Hz}, 1 \mathrm{H}), 7.39(\mathrm{~m}, 3 \mathrm{H}), 7.68(\mathrm{~m}, 2 \mathrm{H}) \cdot-{ }^{13} \mathrm{C} \mathrm{NMR}\left(75 \mathrm{MHz}, \mathrm{CDCl}_{3}\right) \delta=$ 18.2, 20.8, 25.5, 28.2, 28.4, 36.7, 45.5, 68.1, 84.4, 126.7, 128.3, 131.1, 134.8, 166.7. - IR (cm-1, neat) : 3303, 2935, 2859, 1634. - MS (CI:NH$): m / z=244\left[\mathrm{MH}^{+}\right]$. - Anal. Calcd for $\mathrm{C}_{16} \mathrm{H}_{21} \mathrm{NO}$ : C, 78.97; H, 8.70; N, 5.76. Found: C, 78.33; H, 8.66; N, 5.62.

\section{(S)-N-(1-isopropyl-oct-7-ynyl)-benzamide (7b)}

white solid. - M.p. $=64{ }^{\circ} \mathrm{C} .-[\alpha]_{\mathrm{D}}=-14\left(\begin{array}{cc}c & \left.0.95, \mathrm{CHCl}_{3}\right) .\end{array}{ }^{1} \mathrm{H} \mathrm{NMR}\left(300 \mathrm{MHz}, \mathrm{CDCl}_{3}\right): \delta=\right.$ $0.96(\mathrm{~d}, J=6.8 \mathrm{~Hz}, 3 \mathrm{H}), 0.98(\mathrm{~d}, J=6.8 \mathrm{~Hz}, 3 \mathrm{H}), 1.53(\mathrm{~m}, 8 \mathrm{H}), 1.85(\mathrm{~m}, 1 \mathrm{H}), 1.92(\mathrm{t}, J=2.6 \mathrm{~Hz}$, $1 \mathrm{H}), 2.17(\mathrm{td}, J=6.8,2.6 \mathrm{~Hz}, 2 \mathrm{H}), 4.03(\mathrm{~m}, 1 \mathrm{H}) 5.86(\mathrm{~d}, J=9.3 \mathrm{~Hz}, 1 \mathrm{H}), 7.46(\mathrm{~m}, 3 \mathrm{H}), 7.76(\mathrm{~m}$, 2H). - $\left.{ }^{13} \mathrm{C} \mathrm{NMR} \mathrm{(75} \mathrm{MHz,} \mathrm{CDCl}_{3}\right) \delta=17.8,18.3,19.2,25.8,28.3,28.7,32.1,32.3,54.5,68.1$, 84.5, 126.7, 128.6, 131.2, 135.1, 167.3. IR (cm-1, neat) : 3298, 2937, 2857, 1631. - MS (CI: $\left.\mathrm{NH}_{3}\right)$ : $m / z=272\left[\mathrm{MH}^{+}\right]$. Anal. Calcd for $\mathrm{C}_{18} \mathrm{H}_{25} \mathrm{NO}: \mathrm{C}, 79.66 ; \mathrm{H}, 9.28 ; \mathrm{N}, 5.16$. Found: C, 79.38; H, $9.30 ; \mathrm{N}, 4.98$.

\section{(S)-N-(1-phenethyl-oct-7-ynyl)-benzamide (7c)}

white solid. - M.p. $=91{ }^{\circ} \mathrm{C} .-[\alpha] \mathrm{D}=-63.0\left(c 1.00, \mathrm{CHCl}_{3}\right) .-{ }^{1} \mathrm{H}$ NMR $\left(300 \mathrm{MHz}, \mathrm{CDCl}_{3}\right): \delta=$ $1.48(\mathrm{~m}, 8 \mathrm{H}), 1.85(\mathrm{~m}, 2 \mathrm{H}), 1.91(\mathrm{t}, J=2.6 \mathrm{~Hz}, 1 \mathrm{H}), 2.16(\mathrm{td}, J=6.8,2.6 \mathrm{~Hz}, 2 \mathrm{H}), 2.71(\mathrm{t}, J=7.9$ $\mathrm{Hz}, 2 \mathrm{H}), 4.22(\mathrm{~m}, 1 \mathrm{H}) 6.02(\mathrm{~d}, J=9.1 \mathrm{~Hz}, 1 \mathrm{H}), 7.17(\mathrm{~m}, 3 \mathrm{H}), 7.22(\mathrm{~m}, 2 \mathrm{H}), 7.43(\mathrm{~m}, 3 \mathrm{H}), 7.69(\mathrm{~m}$, 2H). ${ }^{13} \mathrm{C} \mathrm{NMR}\left(75 \mathrm{MHz}, \mathrm{CDCl}_{3}\right) \delta=18.2,25.4,28.2,28.6,32.3,35.2,36.9,49.7,68.2,84.5$, 125.8, 131.3, 134.8, 141.8, 167.1. - IR (cm-1, neat) : 3302, 2938, 2856, 2116, 1632. - MS (CI:NH $\left.{ }_{3}\right)$ : $m / z=334\left[\mathrm{MH}^{+}\right]$. Anal. Calcd for $\mathrm{C}_{23} \mathrm{H}_{27} \mathrm{NO}: \mathrm{C}, 82.84 ; \mathrm{H}, 8.16 ; \mathrm{N}, 4.20$. Found: C, 82.76; H, $8.35 ; \mathrm{N}, 4.05$.

\section{2-methyl-4-pentyl-6-phenyl-pyridine (9)}

oil. ${ }^{1} \mathrm{H}$ NMR $\left(300 \mathrm{MHz}, \mathrm{CDCl}_{3}\right): \delta=0.88(\mathrm{t}, J=6.8 \mathrm{~Hz}, 3 \mathrm{H}), 1.31(\mathrm{~m}, 4 \mathrm{H}), 1.62(\mathrm{~m}, 2 \mathrm{H}), 2.57$ $(\mathrm{s}, 3 \mathrm{H}), 2.61(\mathrm{t}, J=7.6 \mathrm{~Hz}, 2 \mathrm{H}), 6.92(\mathrm{~s}, 1 \mathrm{H}), 7.39(\mathrm{~m}, 3 \mathrm{H}), 7.97(\mathrm{~d}, J=7.1 \mathrm{~Hz}, 2 \mathrm{H}) .-{ }^{13} \mathrm{C} \mathrm{NMR}$ $\left(75 \mathrm{MHz}, \mathrm{CDCl}_{3}\right) \delta=14.0,22.5,24.5,30.2,31.4,35.4,118.3,122.0,127.1,128.6,152.9,156.9$, 158.0. - IR (cm-1, neat) : 3038, 2927, 2856, 2232, 1728, 1604. MS (CI:NH $): m / z=240\left[\mathrm{MH}^{+}\right]$. 


\section{Captions for Schemes and Figures}

Scheme 1 : Reagents and conditions: (a) (i) $\mathrm{FcCHO}_{2} \mathrm{NaBH}_{4}, \mathrm{MeOH}$; (ii) $\mathrm{RCHO}, \mathrm{MgSO}_{4}$, THF; (b) PentCCAl $(i-\mathrm{Bu})_{2} . \mathrm{Et}_{3} \mathrm{~N}, \mathrm{AlMe}_{3}$, toluene, $0{ }^{\circ} \mathrm{C}$; (c) $\mathrm{CH}_{2} \mathrm{Cl}_{2}$, TFA $5 \%, \mathrm{Et}_{3} \mathrm{SiH}, 0{ }^{\circ} \mathrm{C}$ to R.T.

Scheme 2 :

Scheme 3 : Reagents and conditions: (a) (i) $\mathrm{H}_{5} \mathrm{IO}_{6}, \mathrm{MeNH}_{2}$; (ii) $\mathrm{HCl} / \mathrm{MeOH}$, (iii) $\mathrm{K}_{2} \mathrm{CO}_{3}$; (b) $\mathrm{PhCOCl}, i-\mathrm{Pr}_{2} \mathrm{EtN}, \mathrm{CH}_{2} \mathrm{Cl}_{2}$.

Scheme 4 :

Scheme 5 :

\section{Acknowledgements}

One of us (J. B.) thanks MENRT for a grant.

\section{References :}

${ }^{[1]}$ For examples of intramolecular hydroaminations of aminoalkynes, see : Y. Li, T. J. Marks, J. Am. Chem. Soc. 1998, 120, 1757-1771 and references therein. For the reactivity of terminal alkynes, see : K. Sonogashira In Comprehensive Organic Synthesis; B. M. Trost, I. Fleming Eds. Pergamon: New-York, 1991; vol. 3, pp. 521-548. (a) C. A. Brown, A. Yamashita J. Am. Chem. Soc. 1975, 97, 891-892. (b) C. A. Brown, A. Yamashita J. Chem. Soc., Chem. Comm., 1976, 959-960.

${ }^{[2]}$ (a) C. A. Brown, A. Yamashita J. Am. Chem. Soc. 1975, 97, 891-892. (b) C. A. Brown, A. Yamashita J. Chem. Soc., Chem. Comm., 1976, 959-960.

${ }^{[3]}$ (a) M. M. Midland, R. L. Halterman, C. A. Brown, A. Yamaichi Tetrahedron Lett. 1981, 39, 4171-4172. (b) M. M. Midland, P. E. Lee J. Org. Chem. 1981, 46, 3933-3934.

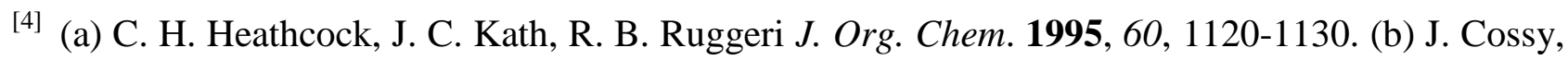
D. Belotti, J.-P. Pete Tetrahedron 1990, 46, 1859-1870.

[5] (a) S. R. Abrams J. Org. Chem. 1984, 49, 3587-3590. (b) W. Fitz, D. Arigoni J. Chem. Soc., Chem. Comm., 1992, 1533-1534.

${ }^{[6]}$ J. Blanchet, M. Bonin, L. Micouin, H.-P. Husson J. Org. Chem. 2000, 65, 6423-6426.

${ }^{[7]}$ J. Cossy, B. Gille, V. Bellosta J. Org. Chem. 1998, 63, 3141-3146.

${ }^{[8]}$ Diastereomeric purity of isomerized compounds has been checked by ${ }^{1} \mathrm{H}$ and ${ }^{13} \mathrm{C}$ NMR.

${ }^{[9]}$ Z.-Y. Chang, R. M. Coates, J. Org. Chem. 1990, 55, 3475-3483.

[10] $\mathrm{H}_{5} \mathrm{IO}_{6}$-mediated oxidative cleavage of similar substrates is known to occur without any racemization : see ref. [9] and [6]. 
${ }^{[11]}$ KAPA has been described to be basic enough to cause $\mathrm{D} / \mathrm{H}$ exchange on benzene- $d_{6}$ : C. A. Brown J. Chem. Soc., Chem. Comm., 1975, 222-223.

[12] The exact mechanism of this unusual cycloaromatization process is unclear. As pointed out by one referee, 9 could result from a possible deprotonation $\alpha$ to the OMe group, leading to a 6-endodig cyclization and aromatization. This explanation implies that several metalated species involved in this process, bearing a $\mathrm{C}-\mathrm{N}$ bond in $\beta$-position, undergo cyclization or protonation faster than $\beta$ elimination. 
